\title{
A 3D flexible neural interface based on a microfluidic interconnection cable capable of chemical delivery
}

\author{
Yoo Na Kang ${ }^{1}$, Namsun Chou $\mathbb{1}^{2}$, Jae-Won Jang ${ }^{3}$, Han Kyoung Choe ${ }^{4}$ and Sohee Kim $\mathbb{1}^{3 凶}$
}

\begin{abstract}
The demand for multifunctional neural interfaces has grown due to the need to provide a better understanding of biological mechanisms related to neurological diseases and neural networks. Direct intracerebral drug injection using microfluidic neural interfaces is an effective way to deliver drugs to the brain, and it expands the utility of drugs by bypassing the blood-brain barrier (BBB). In addition, uses of implantable neural interfacing devices have been challenging due to inevitable acute and chronic tissue responses around the electrodes, pointing to a critical issue still to be overcome. Although neural interfaces comprised of a collection of microneedles in an array have been used for various applications, it has been challenging to integrate microfluidic channels with them due to their characteristic three-dimensional structures, which differ from two-dimensionally fabricated shank-type neural probes. Here we present a method to provide such three-dimensional needle-type arrays with chemical delivery functionality. We fabricated a microfluidic interconnection cable $(\mu \mathrm{FIC})$ and integrated it with a flexible penetrating microelectrode array (FPMA) that has a 3-dimensional structure comprised of silicon microneedle electrodes supported by a flexible array base. We successfully demonstrated chemical delivery through the developed device by recording neural signals acutely from in vivo brains before and after $\mathrm{KCl}$ injection. This suggests the potential of the developed microfluidic neural interface to contribute to neuroscience research by providing simultaneous signal recording and chemical delivery capabilities.
\end{abstract}

\section{Introduction}

With the development of micromachined neural interfaces, various multifunctional neural interfacing systems have been introduced for different applications ${ }^{1-5}$. Multifunctional neural interfaces not only record neural signals but also directly detect electroactive molecules ${ }^{6-8}$, optically stimulate neurons $^{9-11}$, or deliver drugs by integrating microfluidic channels ${ }^{12,13}$. Direct intracerebral drug injection using microfluidic neural interfaces is an effective way to deliver drugs to the brain, as the

Correspondence: Sohee Kim (soheekim@dgist.ac.kr)

${ }^{1}$ Department of Medical Assistant Robot, Korea Institute of Machinery \& Materials (KIMM), Daegu, Republic of Korea

${ }^{2}$ Center for BioMicrosystems, Korea Institute of Science and Technology (KIST), Seoul, Republic of Korea

Full list of author information is available at the end of the article blood-brain barrier (BBB) and blood-cerebrospinal fluid barrier prevent the effective delivery of drugs, including anticancer medicines, antibiotics, and anti-inflammatory reagents, to the brain ${ }^{14}$. To date, a number of microfluidic neural interfaces for direct drug injection have been successfully developed using two-dimensional fabrication processes $^{15-17}$, and the possibility of therapeutic uses to treat brain disorders has been demonstrated ${ }^{18,19}$.

Chen et al. developed a neural probe with multiple flow channels and recorded neural signals evoked by various chemical stimuli in guinea pigs ${ }^{12}$. Takeuchi et al. fabricated a parylene-C (PPX-C)-based flexible neural probe integrated with microfluidic channels ${ }^{20}$, which was inserted into a rat brain with the help of supporting materials such as polyethylene glycol (PEG). Altuna et al. presented a SU-8-based microprobe for neural signal

\section{(c) The Author(s) 2021}

(c) (i) Open Access This article is licensed under a Creative Commons Attribution 4.0 International License, which permits use, sharing, adaptation, distribution and reproduction c. in any medium or format, as long as you give appropriate credit to the original author(s) and the source, provide a link to the Creative Commons license, and indicate if changes were made. The images or other third party material in this article are included in the article's Creative Commons license, unless indicated otherwise in a credit line to the material. If material is not included in the article's Creative Commons license and your intended use is not permitted by statutory regulation or exceeds the permitted use, you will need to obtain permission directly from the copyright holder. To view a copy of this license, visit http://creativecommons.org/licenses/by/4.0/. 
recording and drug delivery ${ }^{21}$. Pongracz et al. developed a 70-mm-long silicon multielectrode integrated with microfluidic channels for deep brain research ${ }^{22}$. Rubehn et al. presented a polyimide-based electrode integrated with a SU-8-based waveguide and a microfluidic chan$\mathrm{nel}^{23}$. In their work, an adeno-associated virus was injected using the microfluidic channel, and the light was transmitted through the waveguide, demonstrating successful optogenetic stimulation and neural signal recording in mice. Lecomte et al. experimented with a PEG and silk fibroin-coated PPX-C neural probe ${ }^{24}$. Lee et al. developed a microdialysis probe for neurochemical monitoring ${ }^{25}$. McCall et al. described a wireless optofluidic neural probe that can be utilized for in vivo pharmacology and optogenetics $^{26}$. Shin et al. demonstrated a multifunctional, multishank neural probe that was integrated with an optical waveguide and microfluidic channels; it covered a wide range, including the hippocampal CA3 to CA1 regions in mice, with no significant tissue damage ${ }^{27}$. The functionality of the probe was confirmed by modulating the functional connections of the hippocampus.

All of these microfluidic neural interfaces have employed shank structures that are fabricated in two-dimensional planes, in which microfluidic channels are integrated horizontally along with the shank. The purposes of drug delivery through such shank-type neural probes have been mainly to provide multifunctional tools for neuroscience research, for instance, for the discovery of neurophysiological and neuropathological mechanisms and neural circuits in small animals ${ }^{28,29}$. To accomplish such purposes, it has been important to establish how many channels can be realized in a small area of the shank and how to deliver drugs to a focused target region precisely ${ }^{12}$.

Unlike the aforementioned shank-type neural probes, there are neural interfaces comprised of a collection of microneedles in an array, including, for example, the Utah electrode array (UEA). The UEA is the only microelectrode array that has been approved by the FDA for use with humans ${ }^{30}$ and has been widely used in large animals such as primates because it contains a large number of electrodes to cover a wide region of the brain ${ }^{31}$. The UEA has a characteristic structure in which microneedles are positioned perpendicularly to the array base, unlike the shank-type probes, in which the electrodes are positioned horizontally along the shank. It has been challenging to integrate additional functions into such silicon-based 3dimensionally fabricated arrays. Therefore, there have been few studies on multifunctional needle-type arrays reported thus $\mathrm{far}^{32,33}$. Abaya et al. introduced an optrode array for infrared neural stimulation ${ }^{34,35}$, which is a rare example of studies integrating additional functions into this needle-type electrode array.

Simeral et al. reported the implantation of UEAs with which neural signals were recorded for 2.7 years in humans ${ }^{36}$. At the end of the study, $\sim 60 \%$ of the electrodes (56 out of 96) detected spike signals. Despite successful long-term recordings, more than $40 \%$ of the electrodes were reported to lose their functionality due to inflammatory responses leading to chronic electrode encapsulation $^{37}$. It has been shown that using shank-type neural probes, the employment of anti-inflammatory drugs such as dexamethasone or neurotrophic medium effectively reduces immunoreactivity during initial immune respon$\operatorname{ses}^{38-40}$. The delivery of anti-inflammatory drugs not only decreases immune responses but also increases the neuronal density around the electrodes ${ }^{41}$. Therefore, adding the capability of drug delivery to the electrodes could improve the lifespans of needle-type neural interfaces, which inevitably involve wounds, or keep more electrodes functional over the entire period of implantation. However, the integration of the drug delivery function into such needle-type microelectrodes has barely been investigated, as it is challenging to integrate a microfluidic channel into the silicon-based 3-dimensional array structure, with needles standing perpendicularly on the wafer surface where fabrication processes are carried out.

Here we present a method for providing threedimensionally fabricated needle-type arrays with chemical delivery functionality for the first time. We previously reported a flexible penetrating microelectrode array (FPMA), which was fabricated three-dimensionally through dicing and etching of bulk silicon from the beginning of fabrication processes; the device was similar in appearance to the UEA but utilized a flexible array base $^{42,43}$. We also reported a flexible interconnection cable used to connect the FPMA with recording equipment $^{42,44,45}$. In the present study, we propose a 3dimensional, flexible, and multifunctional neural interface based on an FPMA integrated with a PPX-C-based microfluidic interconnection cable $(\mu \mathrm{FIC})$ to deliver chemicals to the electrodes (Fig. 1a). The strategy here is to integrate the microfluidic channel into the flexible interconnection cable instead of fabricating the microfluidic channel inside the electrode itself. The fluid flows along the surfaces of needles from the base, resulting in fluid delivery directly to the brain surface but indirectly to the electrodes. The PPX-C-based fabrication of $\mu$ FICs not only maintains the flexibility of the FPMA but also allows freedom in designing the number, shape, and size of microfluidic channels. Using the developed microfluidic neural interface, we demonstrated chemical delivery capability in combination with recording capability through in vivo acute experiments.

\section{Materials and methods \\ Flexible penetrating microelectrode array (FPMA)}

The FPMA used in this study was an array of $4 \times 3$ electrodes with a flexible polydimethylsiloxane (PDMS) 


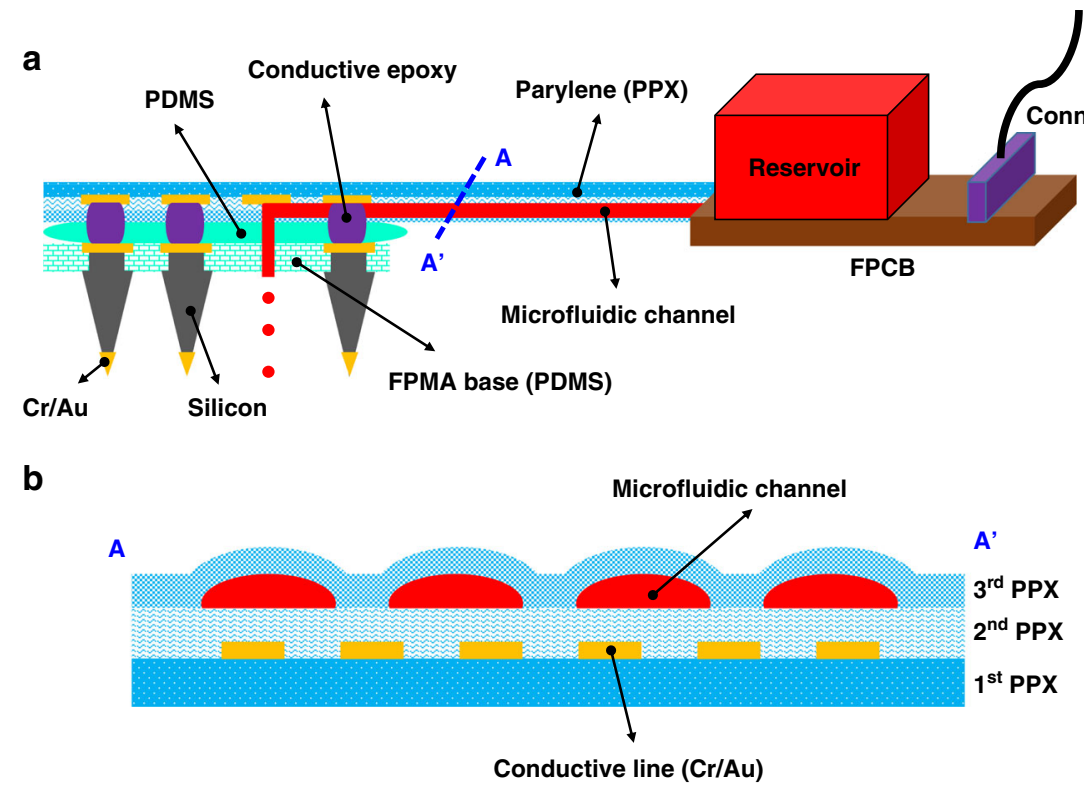

Fig. 1 Schematic illustrations of the microfluidic neural interface. a The flexible penetrating microelectrode array (FPMA) is integrated with the microfluidic interconnection cable $(\mu \mathrm{FIC})$. $\mathbf{b}$ The cross-sectional view of the $\mu \mathrm{FIC}$ (along A-A') shows that it consists of three layers of parylene-C, with embedded conductive lines and microfluidic channel

base. The height of silicon needles and the pitch between them were designed to be 1100 and $550 \mu \mathrm{m}$, respectively. The FPMA was fabricated as reported in the previous studies $^{42,43,46}$. Figure 2a shows the procedures used to fabricate the FPMA and a scanning electron microscopic (SEM) image of the fabricated FPMA. The tips of the silicon needles were coated with $\mathrm{Cr} / \mathrm{Au}$ at thicknesses of 25/200 nm (SRN-110, Sorona, Pyeongtaek, Korea). In addition, an insulation layer of PPX-C was deposited at a thickness of $3 \mu \mathrm{m}$ on the surface of the entire array (NRPC-500, NURI TECH, Incheon, Korea) except for the needle tips ${ }^{44}$. This PPX-C layer helps prevent the silicon needles from being separated from the PDMS base, although the adhesion between the silicon needles and the PDMS base was confirmed previously ${ }^{42}$.

\section{Microfluidic interconnection cable ( $\mu \mathrm{FIC})$}

Figure 1a presents the schematic drawing of the proposed microfluidic neural interface based on the $\mu \mathrm{FIC}$, which consisted of three layers of PPX-C, as detailed in Fig. $1 \mathrm{~b}$. The first layer was the bottom layer serving as the substrate of conductive metal lines, and inlets were located therein. The width and spacing of the conductive metal lines were 90 and $70 \mu \mathrm{m}$, respectively. We created five inlet holes with a diameter of $320 \mu \mathrm{m}$ for secured infusion into the fluidic channel. The second layer was for the insulation of the conductive metal lines and, at the same time, served as the bottom of the microfluidic channel. A microfluidic channel was formed between the second PPX-C layer (insulation layer) and the third PPX-
C layer (microfluidic layer). The $\mu \mathrm{FIC}$ had multiple cratershaped structures designed to prevent the collapse of the third PPX-C layer ${ }^{47}$. These crater-shaped structures on top of the cable, as illustrated in Fig. 1b, were formed by photoresist (PR) patterning on the second PPX-C layer, which was rounded after reflow at a later step, and deposition of the third PPX-C layer. The microfluidic channel was formed where the PR had been after the removal of the PR. The $\mu$ FIC had connection pads on the first PPX-C layer to attach the FPMA and a flexible printed circuit board (FPCB) at each end of the cable, as shown in Fig. 2b-vi.

Figure $2 \mathrm{~b}$ shows the procedures used for the fabrication of the $\mu$ FIC. First, Ti was deposited at a thickness of $200 \mathrm{~nm}$ as a sacrificial layer on a 6-inch silicon wafer using a metal sputtering system (SRN-110, Sorona, Anseong, Korea). The bottom PPX-C layer was deposited at a thickness of $6 \mu \mathrm{m}$ using a low-pressure chemical vapor deposition (LPCVD) system (NRPC-500, NURI$\mathrm{TECH}$, Incheon, Korea) (Fig. 2b-i). Cr/Au with thicknesses of $25 / 200 \mathrm{~nm}$ was deposited and patterned by photolithography and etch-back processes to form conductive lines and pads (Fig. 2b-ii). The 3- $\mu \mathrm{m}$-thick second PPX-C layer was deposited for the insulation of conductive lines and pads. To create inlet holes, a 200-nmthick Ti layer was patterned as a mask on the insulation layer. Oxygen plasma etching was carried out using a reactive ion etching (RIE) system (FabStar, Top Technology, Hwaseong, Korea) with $300 \mathrm{~W}$ power, $100 \mathrm{sccm}$ oxygen flow rate, and 500 mTorr pressure (Fig. 2b-iii). 


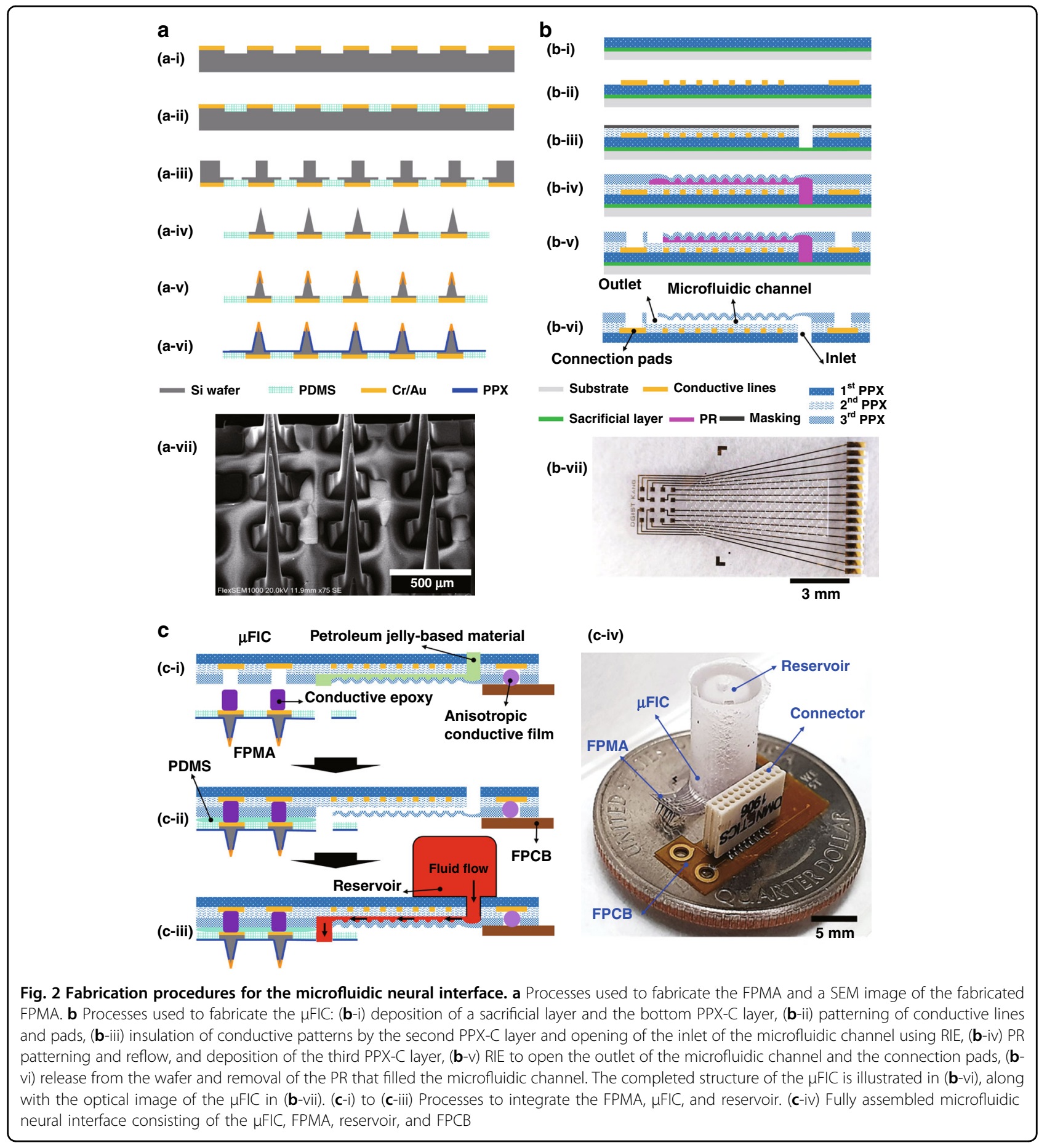

After the removal of the $\mathrm{Ti}$ mask, a photoresist (AZ®40XT, Microchemicals, Ulm, Germany) was patterned on the second PPX-C layer to create the microfluidic channel. The PR pattern was reflowed on a hot plate at $130^{\circ} \mathrm{C}$ to form the arch-shaped fluidic channel and crater-shaped structures, which were removed to create the space through which fluids could flow at the last fabrication step. The top layer of the microfluidic channel was formed by the third PPX-C layer, which was deposited at a thickness of $6 \mu \mathrm{m}$ (Fig. 2b-iv). To expose the pads and to create the outlet of the microfluidic channel, RIE was conducted using $\mathrm{O}_{2}$ plasma with a flow rate of $80 \mathrm{sccm}$, pressure of $200 \mathrm{mTorr}$, and power of $300 \mathrm{~W}$ (Fig. 2b-v). Finally, the $\mu$ FIC was released from the silicon wafer and immersed in acetone and isopropyl alcohol for $5 \mathrm{~min}$ each, to remove the PR pattern that 
filled the inside of the channel (Fig. 2b-vi), resulting in a highly flexible interconnection cable integrated with the arch-shaped microfluidic channel (Fig. 2b-vii).

\section{Assembly and packaging}

The fabricated $\mu$ FIC was assembled with the FPMA, a custom-designed FPCB, and a drug reservoir, as shown in Fig. 2c. The $\mu$ FIC had two sections of connection pads to attach the FPCB and the FPMA at both ends. First, the connection pads on the $\mu \mathrm{FIC}$ and the FPCB were aligned using optical microscopy and connected through an anisotropic conductive film (ACF) (AC-7246LU-18, Hitachi Chemical, Tokyo, Japan) using an ACF bonding machine (ZH-012, Shenzhen ZWX Technology, Guangdong, China) (Fig. 2c-i). The polyimide-based FPCB was used to enable secure connection and disconnection of the $\mu \mathrm{FIC}$ and the recording system. Next, the FPMA was attached to the pads at the other end of the $\mu$ FIC using conductive epoxy (125 epoxy kit, DuralcoTM, New York, NY, USA) (Fig. 2c-i). The epoxy was cured at room temperature for $24 \mathrm{~h}$ to prevent thermal oxidation of the PPX-C-based $\mu \mathrm{FIC}^{48-50}$. Before the attachment of the FPMA, one of the needles on the FPMA was removed by pulling out rapidly with forceps to create the outlet of the microfluidic channel. The place where the needle was removed was aligned to the outlet of the microfluidic channel. After curing the conductive epoxy, PDMS was filled into the space formed between the FPMA and the $\mu$ FIC to maintain the electrical independence of each electrode even in an implanted environment (Fig. 2c-ii). To prevent PDMS from flowing into the outlet while filling the PDMS, the outlet of the $\mu \mathrm{FIC}$ was protected with a petroleum jellybased material (Vaseline ${ }^{\circledR}$, Unilever, London, UK), which melts at body temperature. After the filled PDMS was cured, the outlet of the $\mu \mathrm{FIC}$ was opened again by absorbing the jelly-based material using oil paper in an oven at $37^{\circ} \mathrm{C}$ (Fig. 2c-ii). At the other end of the FPCB, an Omnetics connector (A79018-001, Omnetics, Minneapolis, MN, USA) was soldered, as shown in Fig. 2c-iv.

The drug reservoir was designed to be cylindrical in shape, with a total volume of $\sim 120 \mu \mathrm{l}$. It was made of PDMS and coated with a 3- $\mu$ m-thick layer of PPX-C to prevent the evaporation of the fluid contained in it and to make the surface of the reservoir hydrophobic. To release the internal pressure, a hole with a diameter of $1 \mathrm{~mm}$ was drilled in the top of the reservoir. Before attachment, oxygen plasma was treated to the drug reservoir in order to increase the adhesion between the drug reservoir and the $\mu$ FIC. The drug reservoir was integrated onto the $\mu$ FIC using UV curable epoxy (Fig. 2c-iii). To prevent the flow of UV curable epoxy into the inlet, the microfluidic channel was filled temporarily with PEG, which is solid at room temperature but melts at body temperature. PEG was removed by soaking in deionized water at $37^{\circ} \mathrm{C}$ after the drug reservoir was attached to the $\mu$ FIC. Finally, PDMS was applied to provide mechanical durability and solid electrical insulation at every coupling part where the $\mu \mathrm{FIC}$ and the FPCB were connected and where the Omnetics connector and the FPCB were connected. To protect the fully assembled microfluidic neural interface implanted on an animal's brain, a custom-designed head cap was fabricated with 3D printing using a multi-material rapid prototyping machine (Projet MJP 5500X, 3D systems, Rock Hill, SC, USA), as shown in Fig. 3c.

\section{In vivo experimental procedures}

All experimental procedures were approved by the Institutional Animal Care and Use Committee at the Daegu Gyeongbuk Institute of Science \& Technology (DGIST) Laboratory Animal Resource Center (approval number: DGIST-IACUC-20011506-07). Five adult male Sprague-Dawley rats (Daehan Biolink, Eumseong-gun, Korea) weighing 280-320 g were used. Among the five, three were used for chemical delivery and neural recording experiments, and two were used for histological analysis.

All animals were single-housed under a $12 \mathrm{~h}$ light/dark cycle with free access to food and water and at a controlled temperature of $22-25^{\circ} \mathrm{C}$. The animals were acclimatized to the animal care facility for a week prior to surgery $^{51}$. Before the surgery, the animals were deeply anesthetized using a respiratory anesthetic system (MatrxTM VIP 3000, Midmark, Dayton, OH, USA). Anesthesia was induced in a chamber with $5.0 \%$ isoflurane for $3 \mathrm{~min}$ and maintained with inhalation of $2.0-2.5 \%$ isoflurane through a mask. The animals were mounted on a stereotaxic frame (KOPF \#902, David Kopf Instruments, Tujunga, CA, USA) during the experiment. After incision of the skin, a hole with a diameter of $\sim 3.5 \mathrm{~mm}$ was drilled through the skull for implantation of the fabricated microfluidic neural interface onto the motor cortex. The center of the hole was placed at the primary motor cortex (anterior-posterior (AP): $1.75 \mathrm{~mm}$, medial-lateral (ML): $2.00 \mathrm{~mm}$ from bregma ${ }^{52}$ ). After the dura mater was removed carefully, the electrodes were implanted under instantaneous pressure using a pneumatically actuated impulse inserter (Research inserter IFU, Blackrock ${ }^{\circledR}$ Microsystems, Salt Lake City, UT, USA) to minimize misalignment between the needles of the FPMA in the cortex. The headstage of the recording system was connected with the microfluidic neural interface, and the wand of the pneumatic inserter was mounted on the stereotaxic frame to allow stable operation without shanking. Then, the implanted microfluidic neural interface was secured on the brain using a silicone elastomer (Kwik-Sil, World Precision Instruments, Sarasota, FL, USA) and protected by the head cap fixed on the skull using screws and dental cement, as shown in Fig. 3d. 

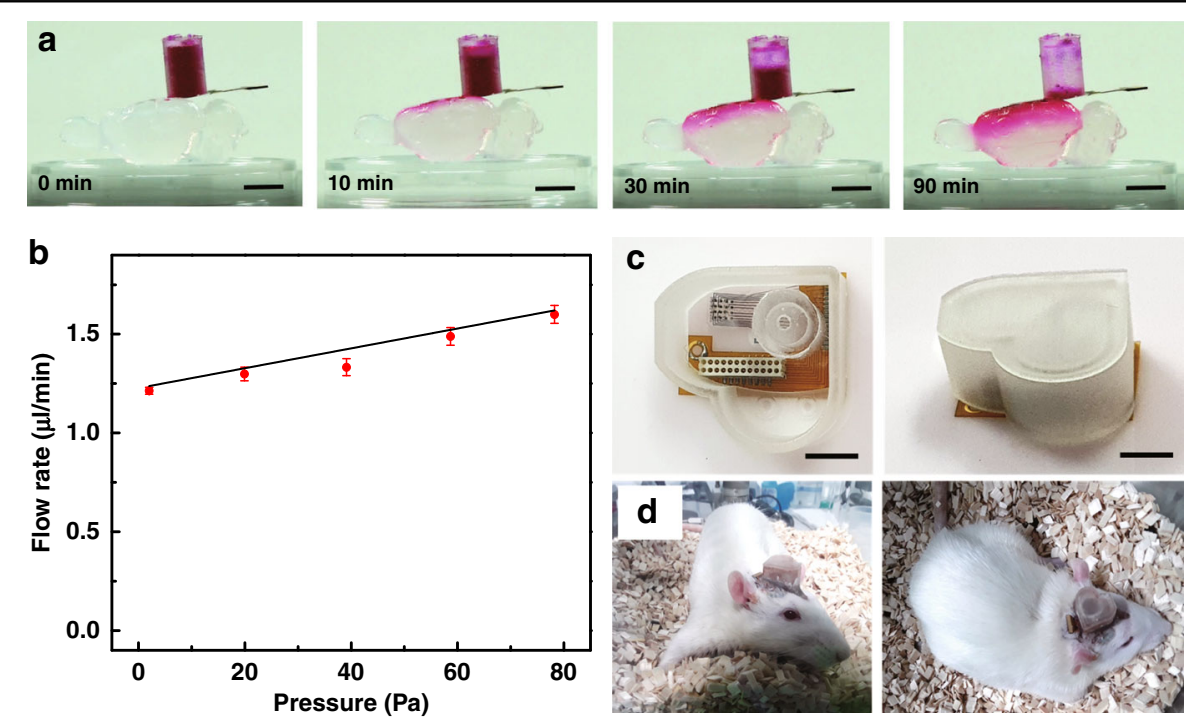

Fig. 3 Fluid delivery through the developed microfluidic neural interface and its implantation in a rat. a Sequential images show the delivery of colored water to a brain model made of $1 \%$ agarose. The colored water diffused into the agarose over time. $\mathbf{b}$ Flow rate through the microfluidic neural interface, as a function of pressure. Red dots and error bars represent means and standard deviations, respectively, while the black line represents linear fitting. Photographs of $\mathbf{c}$ the custom-designed head cap and $\mathbf{d}$ a rat implanted with the microfluidic neural interface protected by the head cap. (scale bar: $5 \mathrm{~mm}$ )

\section{Neural signal acquisition and chemical delivery}

After the surgery, the anesthesia treatment of the rats was maintained. Spontaneous neural signals were recorded at a sampling rate of $30 \mathrm{kHz}$ using a digital recording system (CerePlex Direct, Blackrock ${ }^{\circledR}$ Microsystems, Salt Lake City, UT, USA). Prior to the experiments for fluid delivery, neural signals were recorded using the microfluidic neural interface. First, signals were recorded for $120 \mathrm{~s}$ every $10 \mathrm{~min}$ for a total period of $20 \mathrm{~min}$ without any treatment for baseline. Then, $120 \mu \mathrm{l}$ of $10 \mathrm{mM} \mathrm{KCl}$ in a solution of artificial cerebrospinal fluid (aCSF), prepared following a standard protocol ${ }^{23,53}$, was infused into the reservoir. Neural signals were recorded for $120 \mathrm{~s}$ every $10 \mathrm{~min}$ for a total period of $30 \mathrm{~min}$ immediately after the $\mathrm{KCl}$ solution was injected into the reservoir. After recording, the acquired raw neural signals were bandpass filtered from 250 to $5000 \mathrm{~Hz}$ and used for spike sorting. Blackrock Offline Sorting Software and CerePlex Direct Software (Blackrock ${ }^{\circledR}$ Microsystems, Salt Lake City, UT, USA) were used to detect spikes and calculate signal-tonoise ratios (SNRs). The raw data were then extracted and converted into a format compatible with Spike 2 (Cambridge Electronic Design, Cambridge, UK) by MATLAB (The MathWorks Inc., Natick, MA, USA). Spike sorting and drawing of raster plots were performed using Spike 2. Waveform data were manually discriminated using visual features within $1.6 \mathrm{~ms}$ when the signal amplitude exceeded an adjusted threshold. The extracted waveforms from Spike 2 were plotted again using Origin (OriginLab Co., Northampton, MA, USA).
To verify the region of delivery around the electrodes, microfluidic neural interfaces were implanted into two rats, and Dextran Texas $\operatorname{Red}^{\mathrm{TM}}$ (Invitrogen ${ }^{\mathrm{TM}}$, Waltham, MA, USA) was injected. A total volume of $20 \mu \mathrm{L}$ was injected with a flow rate of 1.2 to $1.5 \mu \mathrm{L} / \mathrm{min}$. After the injection, the animal was left for $10 \mathrm{~min}$ for the fluid to diffuse. Then, the animal was sacrificed and perfused with phosphate-buffered saline (PBS) and 4\% paraformaldehyde (PFA) solution sequentially with a flow rate of $14 \mathrm{~mL} / \mathrm{min}$ for $20 \mathrm{~min}^{54}$. The extracted brain was sliced with a thickness of $100 \mu \mathrm{m}$ using a vibratome (VT1200s, Leica Biosystems, Wetzlar, Germany), and slices were stained with DAPI to label the nuclei of cells.

\section{Results and Discussion}

\section{$\mu F I C$ and fully integrated microfluidic neural interface}

The fabricated $\mu$ FIC is shown in Fig. 4a. The $\mu$ FIC was $13 \mathrm{~mm}$ long, $8 \mathrm{~mm}$ wide, and had multiple crater-shaped structures to support the third layer of PPX-C. The shape of the microfluidic channel depended on the PR pattern created by the reflow process. The PR pattern had multiple holes with a diameter of $200 \mu \mathrm{m}$ and a distance between the centers of $500 \mu \mathrm{m}$. The sharp edges of the PR pattern turned into rounded shapes during the reflow process.

The holes in the PR pattern were deposited with PPX-C, becoming crater-shaped structures in the final form, as shown in Fig. 4b, while the volume occupied by the PR became the inside of the microfluidic channel after 


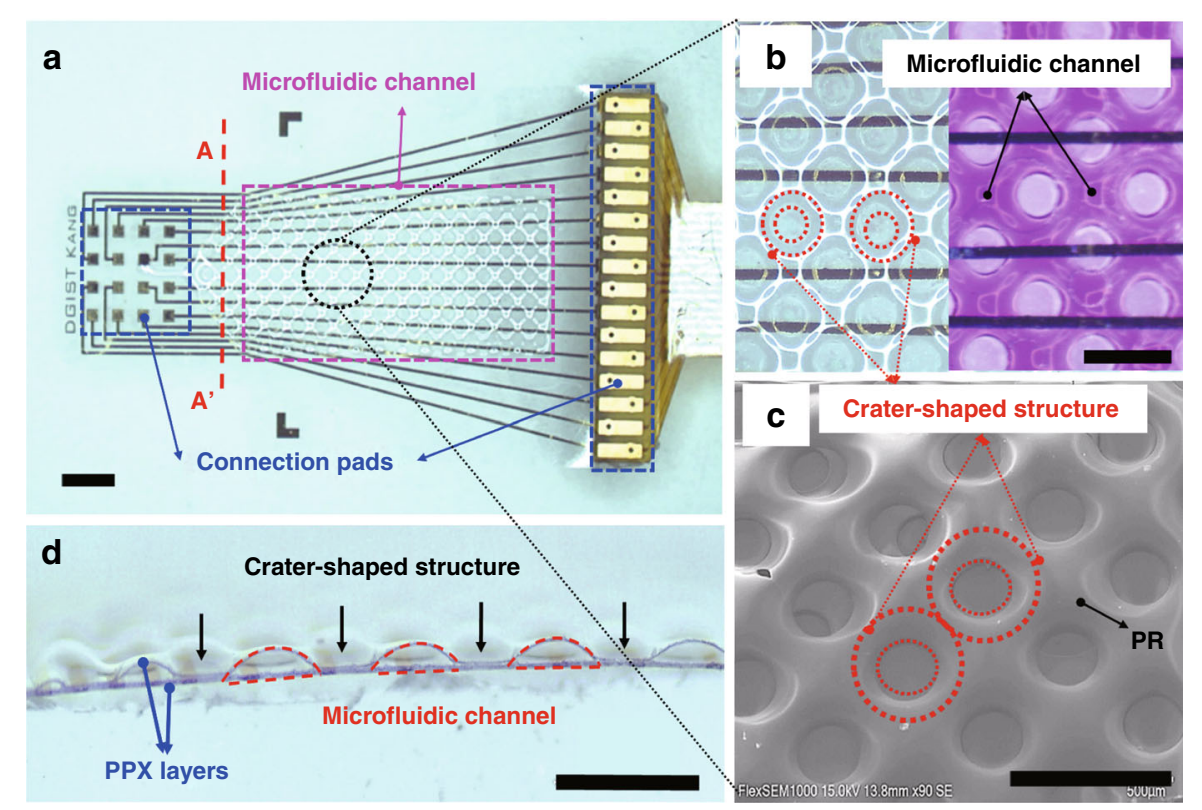

Fig. 4 Optical images of the $\boldsymbol{\mu F I C . ~ a ~ T o p ~ v i e w ~ o f ~ t h e ~} \mu$ FIC. The $\mu$ FIC has a microfluidic channel and connection pads to attach the FPMA (left) and the FPCB (right) (scale bar: $1 \mathrm{~mm}$ ). b The magnified views show when the microfluidic channel is empty (left) and filled with dyed water (right). $\mathbf{c}$ SEM image of the $\mu F I C$. The $\mu F I C$ has crater-shaped structures that help prevent the collapse of the third PPX-C layer. $\mathbf{d}$ Cross-sectional view of the $\mu F I C$ showing the arch-shaped microfluidic channel. The width and height of the channel are $\sim 200$ and $40 \mu \mathrm{m}$, respectively (scale bar: $500 \mu \mathrm{m}$ in b-d)

removal of the PR (Fig. 4c). These crater-shaped structures were located in a zig-zag fashion (Fig. 4b) and thus, helped to avoid collapse or folding of the microfluidic channel by supporting the top PPX-C layer. The cratershaped and zig-zag-placed structures were also used to slow fluid flow in the channel, compared to flow in channels with no such structure or with in-line positioned structures $^{55}$. The cavity generated by removing the PR between the second and third PPX-C layers was the passage through which the fluid flowed. Figure $4 \mathrm{~d}$ shows an optical image of the cross-section of the $\mu$ FIC. The microfluidic channel had arch-shaped cross-sections with a maximum height of $\sim 40 \mu \mathrm{m}$. The number and size of the microfluidic channel depend on the designed PR pattern, which can be easily modified if necessary. Although a microfluidic channel was fabricated in the present study to verify the function of chemical delivery to the FPMA for the first time, it is expected that up to four channels could be fabricated with a minimum channel width of $50 \mu \mathrm{m}$, taking into account the size of the inlet and the entire dimension of the $\mu \mathrm{FIC}$.

To create the outlet, we removed one of the needles, and then, the outlet of the microfluidic channel was aligned at the location from which the needle was removed. Although the outlet was created at the perimeter of the array in the current study, it can be made in the central region as well. The fully integrated microfluidic neural interface, including the FPMA, the $\mu \mathrm{FIC}$, and the reservoir, with dimensions of $12.5 \mathrm{~mm} \times 16 \mathrm{~mm} \times$
$8 \mathrm{~mm}(\mathrm{~W} \times \mathrm{L} \times \mathrm{H})$, is shown in Fig. 2c-iv. The customdesigned head cap, which is shown in Fig. 3c, was used to protect the microfluidic neural interface after implantation in animals.

\section{Characterization of fluid flow through the $\mu$ FIC}

To evaluate fluid delivery through the fabricated $\mu$ FIC, the flow rate was characterized (Fig. 3a). A rat brain model made of $1 \%[\mathrm{w} / \mathrm{v}]$ agarose gel, which was slightly denser than brain tissue, was used ${ }^{56}$. Using dyed water, we observed passive flow into the model brain through diffusion. The flow rate was calculated based on the total volume of the injected fluid and the time taken. Fig. 3b shows the measured flow rate through the $\mu \mathrm{FIC}$, which was $1.40 \pm 0.15 \mu \mathrm{L} / \mathrm{min}$ (mean \pm standard deviation). The maximum allowable flow rate through the $\mu$ FIC was observed to be $5 \mu \mathrm{L} / \mathrm{min}^{57}$. Typically, the total volume of cranial CSF in adult rats is known to be 275 to $440 \mu \mathrm{L}$, and the reproduction rate is 1.77 to $2.84 \mu \mathrm{L} / \mathrm{min}^{58}$. The typical flow rate for direct intra-CSF infusion into the rodent brain is approximately up to $2 \mu \mathrm{L} / \mathrm{min}^{59,60}$. Considering those previous results, the measured passive flow rate was within an acceptable range.

\section{In vivo recording and chemical delivery}

The fabricated microfluidic neural interface was validated through in vivo acute experiments to demonstrate both electrical recording and chemical delivery. We recorded the spontaneous neural activity 


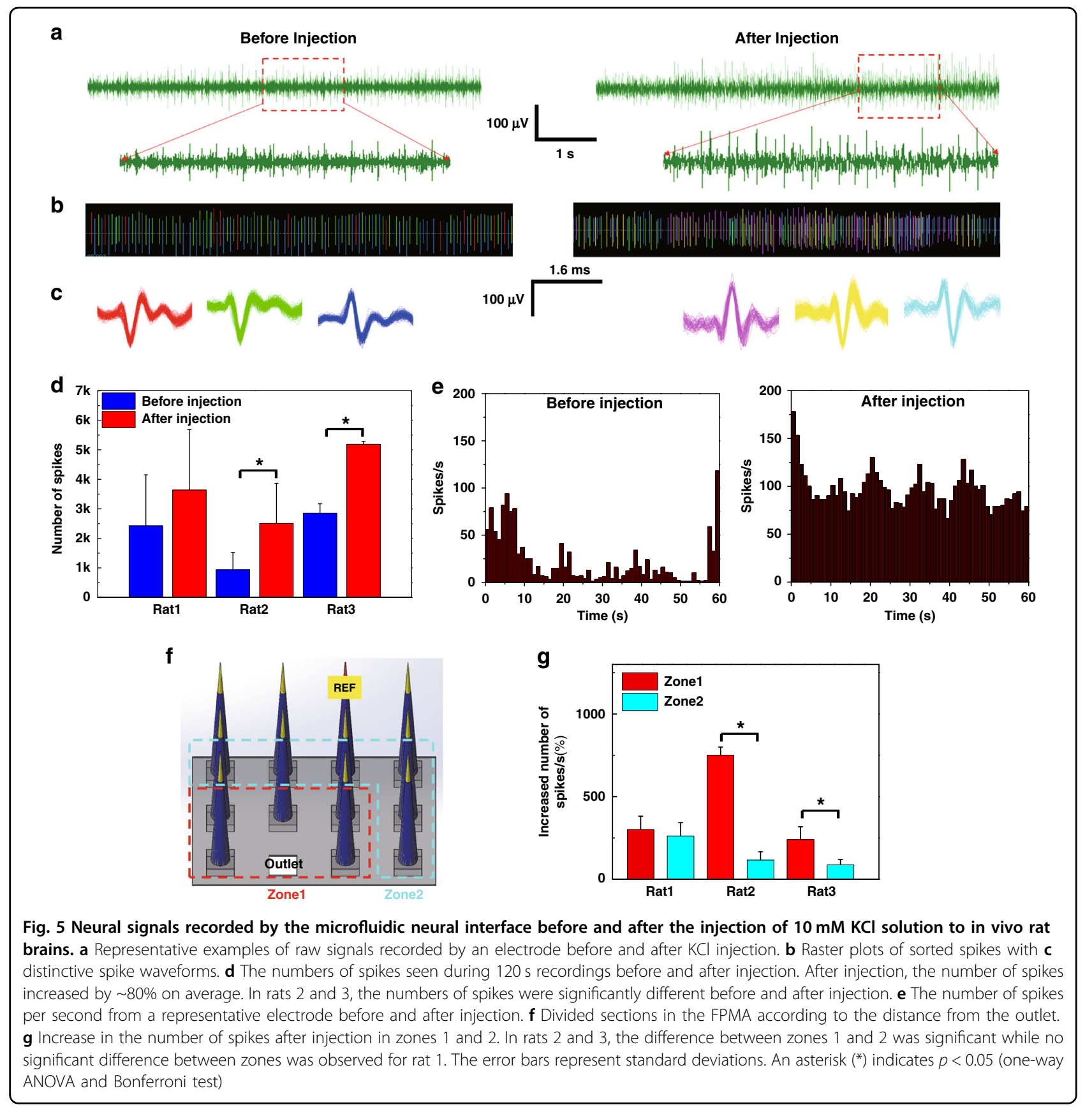

of the primary motor cortex using the device. The in vivo impedance from all 33 electrodes in three FPMAs was measured to be $374.32 \pm 36.86 \mathrm{k} \Omega$ (mean \pm standard deviation). One of the electrodes in each FPMA was assigned as the reference electrode, as shown in Fig. 5f. Spike signals were detected with more than six out of ten electrodes: seven electrodes in rat 1 , eight electrodes in rat 2 , and six electrodes in rat 3. Figure 5a shows representative raw signals obtained by one of the electrodes implanted in rat 2 before and after $\mathrm{KCl}$ injection.
Chemical depolarization typically requires a $20 \mathrm{~min}$ injection of potassium solution, possibly due to the delivery and diffusion required to reach an effective concentration, to induce changes in electric activity. Considering the lag time of the chemical depolarization protocol, signals measured at $30 \mathrm{~min}$ after injection were analyzed. From the 21 functional electrodes in the three implanted arrays, the averaged background noise levels were 43.4 and $37.8 \mu \mathrm{V}_{\mathrm{PP}}$ before and after injection, respectively. Spikes were detected from multiple neurons by a single electrode, with amplitudes of $114.9 \pm 9.4 \mu \mathrm{V}_{\mathrm{PP}}$ 
(mean \pm standard deviation) before injection and $129.7 \pm$ $8.8 \mu \mathrm{V}_{\mathrm{PP}}$ after injection. Therefore, the SNR was calculated to be $2.9 \pm 0.5$ and $3.5 \pm 0.7$ before and after injection, respectively. Figure $5 \mathrm{~b}$ shows the difference between firing rates measured before and after injection. The raster plots derived from spike sorting confirmed that each individual neuron was sorted. In Fig. 5c, the representative waveforms detected by an electrode implanted in rat 2 are compared before and after injection. After injection, the signal amplitude tended to increase slightly, and even changes in waveform were observed, which would indicate that, due to the increased signal amplitude, spike signals from neurons that were not detectable before injection could be detected after injection.

Figure $5 \mathrm{~d}$ shows the number of spikes in three rats before and after injection. The number of spikes detected by each electrode was counted from neural signals recorded for $120 \mathrm{~s}$ at $20 \mathrm{~min}$ before injection and at $30 \mathrm{~min}$ after injection. The bar graph represents the average number of spikes of all electrodes in each array, with error bars indicating standard deviations. In all animals, an increasing tendency in the number of spikes was consistently detected after $\mathrm{KCl}$ injection, as expected. Data were further analyzed by one-way ANOVA followed by the Bonferroni post hoc test to determine statistical significance $(p<0.05)$. In rats 2 and 3 , a significant elevation in the frequency of spikes was observed by all electrodes after injection. Fig. 5e shows a comparison of the numbers of spikes per second before and after injection using one of the electrodes implanted in rat 3 . The number of spikes clearly increased after injection compared to that before injection. Thus, the capability of the developed microfluidic neural interface to deliver chemicals was confirmed by simultaneous recording of neural signals.

Next, the number of spikes as a function of distance from the outlet was compared. The microelectrodes were divided into two sections: electrodes adjacent to the outlet (zone 1) and the rest (zone 2), as shown in Fig. 5f. Figure $5 \mathrm{~g}$ shows an increased percentage in the number of spikes per second after chemical injection. A significant difference $(p<0.05)$ between zones 1 and 2 was observed in rats 2 and 3 . Therefore, it can be concluded that the distancedependent difference in chemical delivery was significant even with passive diffusion-based delivery.

We then histologically examined the range of chemical diffusion around the electrodes. Texas Red-conjugated dextran was injected for 10 min through the outlet located at the base of the microfluidic neural interface to label cells within the effective diffusion range. In Fig. 6, nuclei of cells (blue) and diffused Texas Red-dextran-labeled cells (red) are found in the same area of the brain. The dextran-labeled cells were located within and in close proximity to the cavity of the sharp V-shaped notch formed by microneedle placement, indicating that Texas Red-dextran flowed along the electrodes and diffused into the brain tissue. Histological examinations confirmed that the microfluidic neural interface presented here is capable of delivering chemicals to the electrode sites.

\section{Conclusions}

We presented a three-dimensional flexible and multifunctional neural interface, in which a FPMA was integrated with a $\mu$ FIC. It demonstrated the capability of fluid delivery and simultaneous recording of neural signals in in vivo animals. In the present study, fluid delivery was
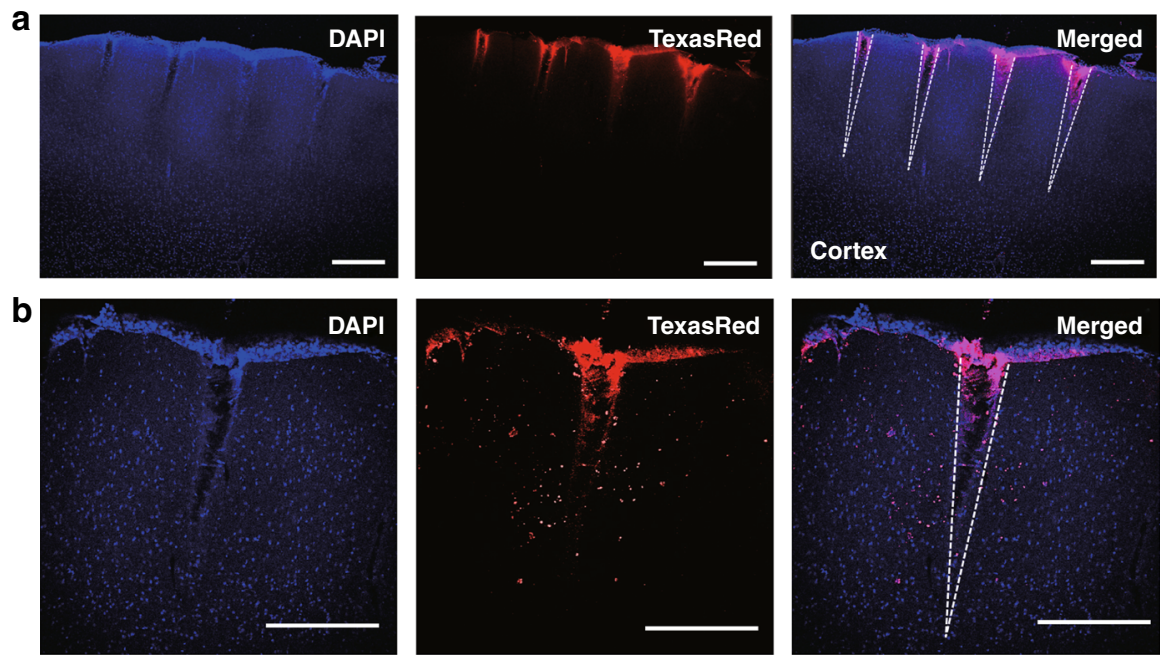

Fig. 6 Histological examination of fluid delivery to the brain in vivo. a Fluorescence images of the rat brain section containing the electrode implantation site. DAPI and Dextran Texas Red were used to label cell nuclei and to visualize the region of delivery, respectively. b Magnified images of the area around an electrode. The white dotted lines represent electrode tracks. (scale bar: $500 \mu \mathrm{m}$ ) 
based on diffusion, and thus, the region more distant from the outlet was influenced less by the delivered chemical, implying that the distant regions would be influenced at a later point in time. To the best of our knowledge, this is the first microfluidic neural interface to add a chemical delivery capability to three-dimensional electrode arrays comprising a collection of microneedles positioned perpendicular to the array base, including the FPMA and the UEA. Moreover, thanks to the flexibility of the device, the developed microfluidic neural interface is expected to cover a large area of the brain even with curvatures, which suggests its potential usage in nonhuman primates and even in humans who have large and convoluted brains. Our study also suggests the possibility of delivering antiinflammatory drugs to needle-type electrode arrays used for long-term implantation. In addition, the successful recording of spike signals before and after chemical delivery indicates that the developed microfluidic neural interface could be used to evaluate drugs to treat brain dysfunctions. As a future study, the delivery of antiinflammatory drugs for a long-term period needs to be investigated.

\section{Acknowledgements}

This work was supported by the Brain Research Program under Grant No. NRF2018M3C7A1022309 through the National Research Foundation of Korea. The authors would like to thank the Center for Core Research Facilities (CCRF) and Laboratory Animal Resource Center (LARC) at DGIST. We also thank Eunju Lee and Gilhyeun Kim (Binaree Inc.) for the perfusion of animals and fluorescence imaging, respectively, and Jihoon Kim (DGIST) for discussions regarding animal experiments.

\section{Author details}

'Department of Medical Assistant Robot, Korea Institute of Machinery \& Materials (KIMM), Daegu, Republic of Korea. ${ }^{2}$ Center for BioMicrosystems, Korea Institute of Science and Technology (KIST), Seoul, Republic of Korea.

${ }^{3}$ Department of Robotics Engineering, Daegu Gyeongbuk Institute of Science and Technology (DGIST), Daegu, Republic of Korea. ${ }^{4}$ Department of Brain \& Cognitive Sciences, Daegu Gyeongbuk Institute of Science and Technology (DGIST), Daegu, Republic of Korea

\section{Author contributions}

Conceptualization: Y.N.K., N.C., and S.K.; Methodology: Y.N.K., N.C., J.-W.J., and H. K.C.; Investigation and formal analysis: Y.N.K.; Validation: Y.N.K., N.C., H.K.C., and S.K.; Resources: H.K.C. and S.K.; Visualization: Y.N.K.; Supervision: S.K.; Writingoriginal draft: Y.N.K.; Writing—review and editing: Y.N.K., H.K.C., and S.K.

\section{Competing interests}

The authors declare no competing interests.

Received: 25 March 2021 Revised: 23 June 2021 Accepted: 11 July 2021 Published online: 18 August 2021

\section{References}

1. Wang, J. et al. A highly selective 3D spiked ultraflexible neural (SUN) interface for decoding peripheral nerve sensory information. Adv. Healthc. Mater. 7, 1700987 (2018).

2. Lee, S. et al. Mechano-neuromodulation of autonomic pelvic nerve for underactive bladder: a triboelectric neurostimulator integrated with flexible neural clip interface. Nano Energy 60, 449-456 (2019).
3. Cho, Y., Park, J., Lee, C. \& Lee, S. Recent progress on peripheral neural interface technology towards bioelectronic medicine. Bioelectron. Med. 6 , 1-10 (2020).

4. Kim, G. H. et al. Recent progress on microelectrodes in neural interfaces. Materials https://doi.org/10.3390/ma11101995 (2018).

5. Chen, R., Canales, A. \& Anikeeva, P. Neural recording and modulation technologies. Nat. Rev. Mater. https://doi.org/10.1038/natrevmats.2016.93 (2017).

6. Zachek, M. K., Park, J., Takmakov, P., Wightman, R. M. \& McCarty, G. S. Microfabricated FSCV-compatible microelectrode array for real-time monitoring of heterogeneous dopamine release. Analyst 135, 1556-1563 (2010).

7. Zachek, M. K., Takmakov, P., Moody, B., Wightman, R. M. \& McCarty, G. S. Simultaneous decoupled detection of dopamine and oxygen using pyrolyzed carbon microarrays and fast-scan cyclic voltammetry. Anal. Chem. 81, 6258-6265 (2009).

8. Vasylieva, N., Marinesco, S., Barbier, D. \& Sabac, A. Silicon/SU8 multi-electrode micro-needle for in vivo neurochemical monitoring. Biosens. Bioelectron. 72, 148-155 (2015)

9. Wu, F. et al. Monolithically integrated $\mu \mathrm{LED}$ s on silicon neural probes for highresolution optogenetic studies in behaving animals. Neuron $\mathbf{8 8}, 1136-1148$ (2015).

10. Park, S. et al. One-step optogenetics with multifunctional flexible polymer fibers. Nat. Neurosci. 20, 612-619 (2017).

11. Goncalves, S. B. et al. LED optrode with integrated temperature sensing for optogenetics. Micromachines https://doi.org/10.3390/mi9090473 (2018).

12. Chen, J., Wise, K. D., Hetke, J. F. \& Bledsoe, S. C. A multichannel neural probe for selective chemical delivery at the cellular level. IEEE Trans. Biomed. Eng. 44, 760-769 (1997).

13. Lee, W. H., Slaney, T. R., Hower, R. W. \& Kennedy, R. T. Microfabricated sampling probes for in vivo monitoring of neurotransmitters. Anal. Chem. 85, 3828-3831 (2013).

14. Kaurav, H. \& Kapoor, D. N. Implantable systems for drug delivery to the brain Ther. Deliv. 8, 1097-1107 (2017).

15. Spieth, S. et al. A floating 3D silicon microprobe array for neural drug delivery compatible with electrical recording. J. Micromech. Microeng. https://doi.org/ 10.1088/0960-1317/21/12/125001 (2011)

16. Wen, X., Liu, T. \& Chiou, P. -Y. A hybrid silicon-PDMS multifunctional neural probe. In Proc. 2016 International Conference on Optical MEMS and Nanophotonics. 1-2 (2016).

17. Liu, B. et al. Enhanced biocompatibility of neural probes by integrating microstructures and delivering anti-inflammatory agents via microfluidic channels. J. Neural Eng. https://doi.org/10.1088/1741-2552/aa52dc (2017).

18. Slezia, A., Proctor, C. M., Kaszas, A., Malliaras, G. G. \& Williamson, A. Electrophoretic delivery of $\gamma$-aminobutyric acid (GABA) into epileptic focus prevents seizures in mice. J. Vis. Exp. 2019, 1-9 (2019).

19. Proctor, C. M. et al. Electrophoretic drug delivery for seizure control. Sci. Adv. https://doi.org/10.1126/sciadv.aau1291 (2018).

20. Takeuchi, S., Ziegler, D., Yoshida, Y., Mabuchi, K. \& Suzuki, T. Parylene flexible neural probes integrated with microfluidic channels. Lab Chip. 5, 519-523 (2005).

21. Altuna, A. et al. SU-8 based microprobes for simultaneous neural depth recording and drug delivery in the brain. Lab Chip. 13, 1422-1430 (2013).

22. Pongrácz, A. et al. Deep-brain silicon multielectrodes for simultaneous in vivo neural recording and drug delivery. Sens. Actuators B Chem. 189, 97-105 (2013).

23. Rubehn, B., Wolff, S. B. E., Tovote, P., Luthi, A. \& Stieglitz, T. A polymer-based neural microimplant for optogenetic applications: design and first in vivo study. Lab Chip. 13, 579-588 (2013).

24. Lecomte, A. et al. Silk and PEG as means to stiffen a parylene probe for insertion in the brain: toward a double time-scale tool for local drug delivery. J. Micromech. Microeng. https://doi.org/10.1088/0960-1317/25/12/125003 (2015).

25. Lee, W. H. et al. Microfabrication and in vivo performance of a microdialysis probe with embedded membrane. Anal. Chem. 88, 1230-1237 (2016).

26. McCall, J. G. et al. Preparation and implementation of optofluidic neural probes for in vivo wireless pharmacology and optogenetics. Nat. Protoc. 12, 219-237 (2017).

27. Shin, $H$. et al. Multifunctional multi-shank neural probe for investigating and modulating long-range neural circuits in vivo. Nat. Commun. https://doi.org/ 10.1038/s41467-019-11628-5 (2019).

28. Kohl, M. M. et al. Hemisphere-specific optogenetic stimulation reveals left-right asymmetry of hippocampal plasticity. Nat. Neurosci. 14, 1413 (2011). 
29. Shankar, G. M. et al. Amyloid-beta protein dimers isolated directly from Alzheimer's brains impair synaptic plasticity and memory. Nat. Med. 14, 837-842 (2008).

30. Leber, M. et al. Advances in penetrating multichannel microelectrodes based on the Utah array platform. Neural Interface Front. Appl. 1101, 1-40 (2019).

31. Choi, J. R., Kim, S. M., Ryu, R. H., Kim, S. P. \& Sohn, J. W. Implantable neural probes for brain-machine interfaces - Current developments and future prospects. Exp. Neurobiol. 27, 453-471 (2018).

32. Xiang, Z., Wang, H., Pastorin, G. \& Lee, C. Development of a flexible and disposable microneedle-fluidic-system with finger-driven drug loading and delivery functions for inflammation treatment. J. Microelectromech. Syst. 24, 565-574 (2015)

33. Wang, H., Pastorin, G. \& Lee, C. Toward self-powered wearable adhesive skin patch with bendable microneedle array for transdermal drug delivery. Adv. Sci. 3, 1500441 (2016)

34. Abaya, T. V. F. et al. Characterization of a $3 D$ optrode array for infrared neural stimulation. Biomed. Opt. Express 3, 2200-2219 (2012).

35. Abaya, T. V. F. et al. Deep-tissue light delivery via optrode arrays. J. Biomed. Opt. 19, 015006 (2014)

36. Simeral, J. D., Kim, S. P., Black, M. J., Donoghue, J. P. \& Hochberg, L. R. Neural control of cursor trajectory and click by a human with tetraplegia 1000 days after implant of an intracortical microelectrode array. J. Neural Eng. https://doi. org/10.1088/1741-2560/8/2/025027 (2011).

37. Kook, G., Lee, S. W., Lee, H. C., Cho, I. J. \& Lee, H. J. Neural probes for chronic applications. Micromachines https://doi.org/10.3390/mi7100179 (2016).

38. Mercanzini, A. et al. Controlled release nanoparticle-embedded coatings reduce the tissue reaction to neuroprostheses. J. Control. Release 145, 196-202 (2010).

39. De la Oliva, N., Navarro, X. \& del Valle, J. Dexamethasone reduces the foreign body reaction to intraneural electrode implants in the peripheral nerve of the rat. Anat. Rec. 301, 1722-1733 (2018).

40. Kato, Y., Saito, I., Hoshino, T., Suzuki, T. \& Mabuchi, K. Preliminary study of multichannel flexible neural probes coated with hybrid biodegradable polymer. Conf. Proc. IEEE Eng. Med. Biol. Soc. https://doi.org/10.1109/ IEMBS.2006.259978 (2006).

41. Huang, W. C. et al. Multifunctional 3D patternable drug-embedded nanocarrier-based interfaces to enhance signal recording and reduce neuron degeneration in neural implantation. Adv. Mater. 27, 4186-4193 (2015).

42. Byun, D., Cho, S. J. \& Kim, S. Fabrication of a flexible penetrating microelectrode array for use on curved surfaces of neural tissues. J. Micromech. Microeng. https://doi.org/10.1088/0960-1317/23/12/125010 (2013).
43. Byun, D. et al. Recording nerve signals in canine sciatic nerves with a flexible penetrating microelectrode array. J. Neural Eng. https://doi.org/10.1088/17412552/aa7493 (2017).

44. Kang, Y. N. et al. An intrafascicular neural interface with enhanced interconnection for recording of peripheral nerve signals. IEEE Trans. Neural Syst. Rehabil. Eng. 27, 1312-1319 (2019).

45. Oh, K., Byun, D. \& Kim, S. Polymer-based interconnection cables to integrate with flexible penetrating microelectrode arrays. Biomed. Microdevices. 19, 76 (2017).

46. Chou, N. et al. Development of an intrafascicular neural interface for peripheral nerve implantation. IEEE Int. Conf. Rehabil. Robot. https://doi.org/10.1109/ ICORR.2017.8009354 (2017).

47. Huang, Y. G. Y. et al. Stamp collapse in soft lithography. Langmuir 21 8058-8068 (2005).

48. Grattan, D. W. \& Bilz, M. The thermal aging of parylene and the effect of antioxidant. Stud. Conserv. 36, 44-52 (1991).

49. Nowlin, T. E., Smith, D. F. Jr \& Cieloszyk, G. S. Thermal oxidative stability of polyp-xylylenes. J. Polym. Sci. Polym. Chem. Ed. 18, 2103-2119 (1980).

50. Kim, B. J. \& Meng, E. Micromachining of parylene C for bioMEMS. Polym. Adv. Technol. 27, 564-576 (2016).

51. Ma, S. et al. Nucleus incertus promotes cortical desynchronization and behavioral arousal. Brain Struct. Funct. 222, 515-537 (2017).

52. Paxinos, G. \& Watson, C. The Rat Brain in Stereotaxic Coordinates: Hard Cover Edition (Elsevier, 2006).

53. Zhang, J. M. et al. The endocannabinoid system contributes to memory deficits induced by rapid-eye-movement sleep deprivation in adolescent mice. Neuroscience 433, 174-183 (2020).

54. Gage, G. J., Kipke, D. R. \& Shain, W. Whole animal perfusion fixation for rodents. J. Vis. Exp. https://doi.org/10.3791/3564 (2012).

55. Salim, A. M. V., Arumugham, B. \& Madhu, K. G. Improved mixing efficiency and scaling of microfluidic mixer for miRNA based diagnostics. Biotechnol. Indian J. 13, 1-12 (2017)

56. Chen, Z.-J. et al. A realistic brain tissue phantom for intraparenchymal infusion studies. J. Neurosurg. 101, 314-322 (2004).

57. Kang, Y. N., Jang, J. \& Kim, S. Proc. 40th International Conference of the IEEE Engineering in Medicine and Biology Society (IEEE, 2018).

58. Chiu, C. et al. Temporal course of cerebrospinal fluid dynamics and amyloid accumulation in the aging rat brain from three to thirty months. Fluids Barriers CNS. 9, 3 (2012).

59. Ding, G. L. et al. MRI investigation of glymphatic responses to Gd-DTPA infusion rates. J. Neurosci. Res. 96, 1876-1886 (2018).

60. Shin, H. et al. Neural probes with multi-drug delivery capability. Lab Chip. 15 3730-3737 (2015). 